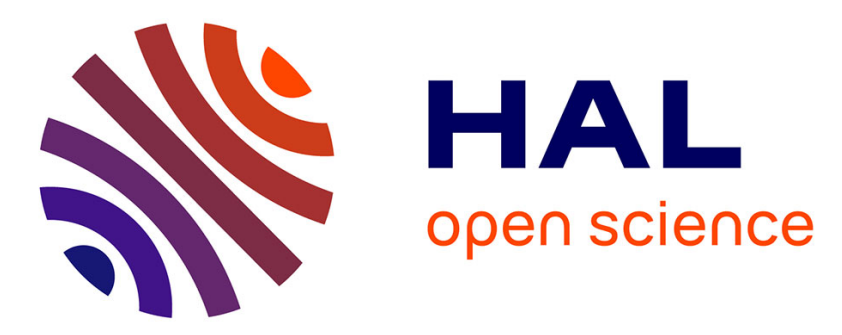

\title{
Coupled electromagnetic modes between a corrugated surface and a thin probe tip \\ Christian Girard, X. Bouju
}

\section{To cite this version:}

Christian Girard, X. Bouju. Coupled electromagnetic modes between a corrugated surface and a thin probe tip. Journal of Chemical Physics, 1991, 95 (3), pp.2056-2064. 10.1063/1.461005 . hal01795481

\section{HAL Id: hal-01795481 \\ https://hal.science/hal-01795481}

Submitted on 18 May 2018

HAL is a multi-disciplinary open access archive for the deposit and dissemination of scientific research documents, whether they are published or not. The documents may come from teaching and research institutions in France or abroad, or from public or private research centers.
L'archive ouverte pluridisciplinaire HAL, est destinée au dépôt et à la diffusion de documents scientifiques de niveau recherche, publiés ou non, émanant des établissements d'enseignement et de recherche français ou étrangers, des laboratoires publics ou privés. 


\title{
Coupled electromagnetic modes between a corrugated surface and a thin probe tip
}

\author{
C. Girard and X. Bouju \\ Laboratoire de Physique Moléculaire-UA CNRS 772-Université de Franche-Comté-25030 Besancon \\ Cedex France
}

(Received 4 January 1991; accepted 19 April 1991)

\begin{abstract}
A self-consistent formalism is presented in order to determine the dispersion equation of the coupled electromagnetic modes between a dielectric probe tip of arbitrary shape and a rough surface. A microscopic picture of the matter is used for describing the dielectric response of the tip, and the coupling with the substrate is introduced from a dynamical matrix expressed in terms of propagators. Retardation effects may be included without formal difficulty through an appropriate response function describing the surface of the solid (local or nonlocal). An advantage of such a calculation lies in the possibility of simulating dielectric tips of arbitrary shape without introducing boundary conditions at the surface of the probe. Connection with atomic force microscopy and near field detection by local probe will be discussed.
\end{abstract}

\section{INTRODUCTION}

The interpretation of recent experimental studies performed near corrugated surfaces from devices using local probe detection, represents a fascinating subject. In fact, since Binnig and Rohrer ${ }^{1}$ demonstrated that it was possible to move in a controlled way a very thin metallic tip at some Angstroms from a surface, a new physics was born and many other related techniques ${ }^{2,3}$ have been developed. Moreover, other devices using local scanning probe detection have revealed interesting behaviors (localized resonances, ${ }^{4}$ polarization effects, ${ }^{5}$ electroluminescence phenomena, ${ }^{6} .$. ) and specific results have been obtained on many substrates. In particular, the concept of using a force at the atomic scale ${ }^{3}$ for imaging the topography of a surface, appears as a complementary tool of the scanning tunneling microscopy (STM) for observing the surface of dielectric samples. The interpretation of atomic and nanometer size resolution in atomic force microscopy ${ }^{7-14}$ (AFM) depends strongly on the tip-sample interaction and various theoretical models have been already proposed. ${ }^{15-23}$

In scanning near-field optical microscopy (SNOM), ${ }^{24}$ the radiation from a nanodetector (pointed optical fiber, ${ }^{25-28}$ metallic subwavelength particle, ${ }^{4} \ldots$ ) is analyzed for different detector-sample positions and images with a resolution far beyond the diffraction limit have been obtained. Recent progresses in this field have thus stimulated some theoretical calculations in order to interpret the relation existing between the image and the object. ${ }^{29-31} \mathrm{~A}$ detailed analysis of physical mechanisms appearing in SNOM and in atomic force microscopy seems to indicate that the detector perturbs the field generated by the isolated surface in a significant way. In other words, the probe tip and the sample interact very strongly and the problem can only be treated by regarding them as forming a single system.

There are various ways for determining the eigenfrequencies of a coupled system. Maxwell equations can be solved in some specific cases where the boundary conditions can be easily defined. In this context Pohl et al. ${ }^{31}$ have pre- sented an exact solution of Laplace equation for an hyperboloid tip interacting with a planar surface. From this continuous approach these authors have found electric field highly localized in the gap region for frequency close to that of the resonant modes of the system. A different method based on a microscopic description of the matter constituting the detector may be considered. In this case, one assumes a discrete distribution of elementary microsystems (atoms, small polarizable spheres,...) characterized by a local response function. A first model has been recently developed in this context in view to understand the relation between the image and the object in scanning tunneling optical microscopy ${ }^{30}$ (STOM). In this work, ${ }^{30}$ the detector was described as a small dielectric sphere treated within the continuous approximation whereas surface corrugations were modeled as a set of polarizable atoms.

In this paper, we wish to show that the coupled modes method can provide a convenient framework for describing the behavior of certain observables measured in local probe microscopy (dispersion force, scattered intensity, polarization effects,...). Note that since the first experimental studies concerned with surface enhanced Raman scattering, many similar calculations have been developed to explain the dynamical behavior of molecules or spherical particles interacting with rough surfaces. ${ }^{32-42}$ In the present work such standard methods will be applied in order to present a tractable formalism leading to a realistic description of the detection processes by taking into account the perturbation induced by the surface from a propagator mediating the information between each part of the tip and the sample. Thus all correlations inside the detector and the surface will be included from a dynamical matrix allowing to obtain the eigenfrequencies of the whole system. As expected, the principal advantage of such a calculation lies in the possibility of simulating a dielectric tip of arbitrary shape without introducing complexity due to boundary conditions. Nevertheless, one must keep in mind that such an advantage is associated with a new computational difficulty connected to the great dimension of the dynamical matrix. However, in most 


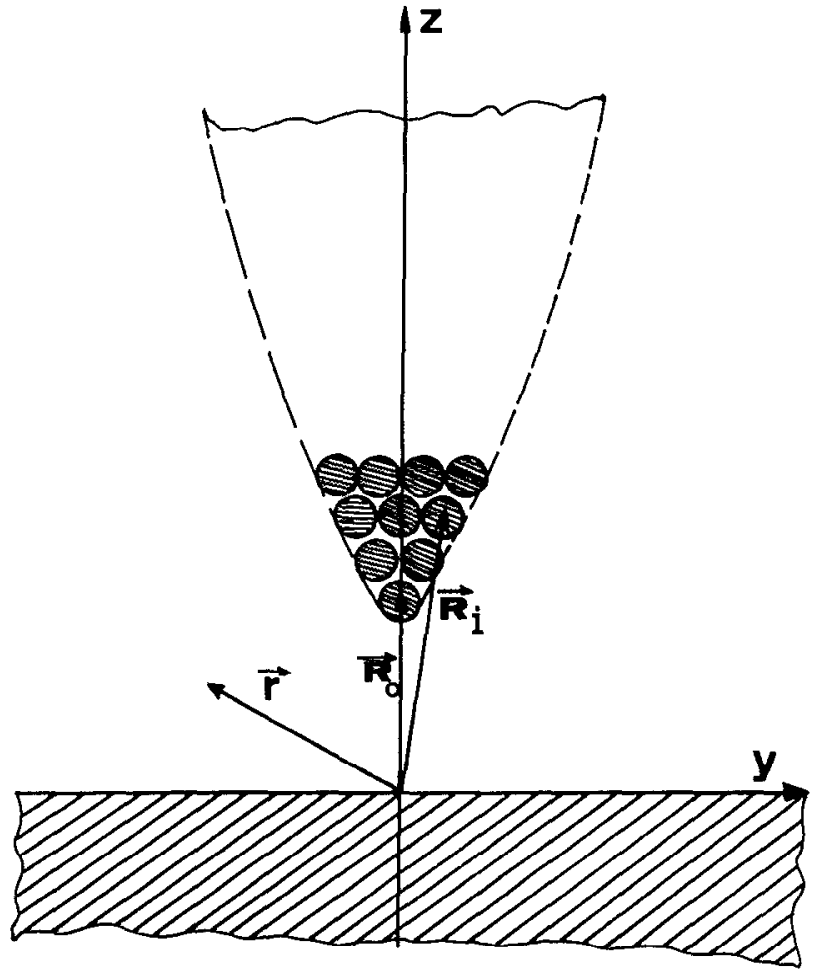

FIG. 1. Geometry of the subwavelength detector interacting with a solid surface. The vector $\mathbf{R}_{0}=\left(0,0, Z_{0}\right)$ represents the position of the atom placed at the apex of the tip. Other atoms are located at the position $\mathbf{R}_{t}$ with respect to the absolute frame of the surface.

cases, we will show that it is not necessary to invert this matrix and that an expansion may be sufficient (nonresonant processes).

The paper will be organized as follows. In Sec. II, we define the geometry of the system and the self-consistent condition is written. Different forms of dipolar propagators are discussed in Sec. III and connections with experimental situations will be analyzed in Secs. IV and V.

\section{THE SELF-CONSISTENT CONDITION}

We consider here the problem of a pointed dielectric stylus placed in front of a surface (dielectric or metallic) as shown in Fig. 1. Moreover, we assume that the tip may be described as a set of polarizable microsystems characterized by a dynamical linear polarizability $\boldsymbol{\alpha}_{i}(\omega)$. We calculate then the effective electric field acting on each polarizable system when the detector is submitted to an external field $\mathbf{E}_{0}(r, \omega)$ of arbitrary spatial dependence. Inside the matter, each microsystem will play the role of a dipolar scattering center excited by the external field $\mathbf{E}_{0}\left(\mathbf{R}_{i}, \omega\right)$ (where $\mathbf{R}_{i}$ represents the position vector of the $i$ th microsystem with respect to the absolute frame).

\section{A. The effective field inside the detector}

In the absence of surface, the microscopic components acquire a fluctuating dipole moment which is modified by the dielectric surrounding generated by other induced dipole moments. In order to take into account the presence of the

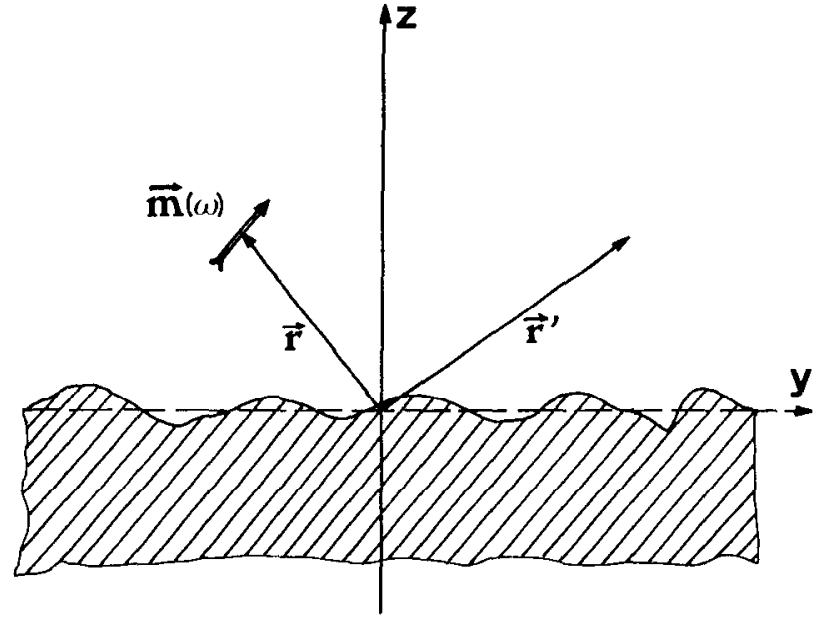

FIG. 2. A fluctuating dipole moment placed in front of a rough surface, $r$ defines the location of the dipole.

sample, it is necessary to introduce the feedback effects due to a polarization of the surface induced by each scattering center. This may be performed by introducing the concept of field propagator. Such a quantity noted $\mathbf{S}\left(\mathbf{r}, \mathbf{r}^{\prime}, \omega\right)$ is defined by the following linear relation: ${ }^{43-49}$

$$
\mathbf{E}\left(\mathbf{r}^{\prime}, \omega\right)=\mathbf{S}\left(\mathbf{r}^{\prime}, \mathbf{r}, \omega\right) \cdot \mathbf{m}(\omega)
$$

and represents the dipolar propagator giving the electric field $\mathbf{E}\left(\mathbf{r}^{\prime}, \omega\right)$ at $\mathbf{r}^{\prime}$ caused by an arbitrary dipole $\mathbf{m}(\omega)$ located at $\mathbf{r}$ (Fig. 2). This key quantity of our problem allows us to write a self-consistent equation for the effective electric field acting on each microscopic component belonging to the detector:

$$
\begin{aligned}
\mathbf{E}\left(\mathbf{R}_{i}, \omega\right)= & \mathbf{E}_{0}\left(\mathbf{R}_{i}, \omega\right)+\sum_{j=1}^{n} \mathbf{S}\left(\mathbf{R}_{i}, \mathbf{R}_{j}, \omega\right) \cdot \boldsymbol{\alpha}_{j}(\omega) \cdot \mathbf{E}\left(\mathbf{R}_{j}, \omega\right) \\
& +\sum_{j \neq i} \mathbf{T}\left(\mathbf{R}_{i j}, k_{0}\right) \cdot \boldsymbol{\alpha}_{j}(\omega) \cdot \mathbf{E}\left(\mathbf{R}_{j}, \omega\right)
\end{aligned}
$$

in which $\mathbf{E}\left(\mathbf{R}_{i}, \omega\right)$ defines the effective field at the $i$ th site and $\mathbf{T}$ labels the dipolar propagator in the vacuum:

$$
\begin{aligned}
\mathbf{T}\left(\mathbf{R}_{i j}, k_{0}\right)= & \exp \left[-i k_{0} R_{i j}\right]\left[\mathbf{T}_{3}\left(\mathbf{R}_{i j}\right)\right. \\
& \left.+i k_{0} \mathbf{T}_{2}\left(\mathbf{R}_{i j}\right)-k_{0}^{2} T_{1}\left(\mathbf{R}_{i j}\right)\right],
\end{aligned}
$$

where $k_{0}$ is the wave vector in the vacuum, $\mathbf{R}_{i j}=\mathbf{R}_{i}-\mathbf{R}_{j}$ and the second rank tensors $\mathbf{T}_{1}, \mathbf{T}_{2}$, and $\mathbf{T}_{3}$ are given by

$$
\begin{aligned}
& \mathbf{T}_{1}(\mathbf{r})=\left[\mathbf{r r}-r^{2} \mathbf{I}\right] r^{-3}, \\
& \mathbf{T}_{\alpha}(\mathbf{r})=\left[3 \mathbf{r r}-r^{2} \mathbf{I}\right] r^{-(\alpha+2)}, \quad \alpha=(2,3),
\end{aligned}
$$

where I represents the identity tensor. Moreover, the third term in the right-hand side of Eq. (2) introduces the dipolar correlations inside the detector. When the spatial extension of the tip is small with respect to the wavelength $\lambda=2 \pi / k_{0}$ of the external field, the two retarded contributions associated to $\mathbf{T}_{2}$ and $\mathbf{T}_{3}$ can be neglected. 
Now in order to solve Eq. (2) it is convenient to introduce two supervectors defined by

$$
\mathbf{F}(\omega)=\left[\mathbf{E}\left(\mathbf{R}_{1}, \omega\right), \mathbf{E}\left(\mathbf{R}_{2}, \omega\right), \ldots, \mathbf{E}\left(\mathbf{R}_{n}, \omega\right)\right]
$$

and

$$
\mathbf{B}(\omega)=\left(\begin{array}{ccc}
\boldsymbol{\alpha}_{1} \cdot \mathbf{S}_{1,1} & \boldsymbol{\alpha}_{2} \cdot\left(\mathbf{T}_{1,2}+\mathbf{S}_{1,2}\right) & \cdots \\
\boldsymbol{\alpha}_{1} \cdot\left(\mathbf{T}_{2,1}+\mathbf{S}_{2,1}\right) & \boldsymbol{\alpha}_{2} \cdot \mathbf{S}_{22} & \\
\cdot & & \\
\cdot & & \\
\cdot & & -
\end{array}\right.
$$

in which one has put $\boldsymbol{\alpha}_{i} \equiv \boldsymbol{\alpha}_{i}(\omega), \mathbf{T}_{i j} \equiv \mathbf{T}\left(\mathbf{R}_{i}-\mathbf{R}_{j}, k_{0}\right)$ and $\mathbf{S}_{i j} \equiv \mathbf{S}\left(\mathbf{R}_{i}, \mathbf{R}_{j}, \omega\right)$. The diagonal terms of $\mathbf{B}(\omega)$ describe the direct tip-sample coupling while the off-diagonal terms are responsible for the substrate mediated interactions inside the system. The solution of the nonhomogeneous self-consistent Eq. (8) can be written as a very compact form,

$$
\mathbf{F}(\omega)=[\mathbf{I}-\mathbf{B}(\omega)]^{-1} \cdot \mathbf{F}_{0}(\omega) .
$$

Thus from the knowledge of the field distribution $\mathbf{E}_{0}\left(\mathbf{R}_{i}, \omega\right)$ this relation allows us to determine the true field in any point inside the detector.

\section{B. The coupled modes of the tip-sample system}

Now if the source field contained in the supervector $\mathbf{F}_{0}(\omega)$ vanishes, we obtain the following eigenvalue equation:

$$
\mathbf{F}(\omega)=\mathbf{B}(\omega) \cdot \mathbf{F}(\omega) \text {. }
$$

Consequently, the allowed coupled modes are given by the solutions of the dispersion equation

$$
D(\omega)=\operatorname{det}[\mathbf{I}-\mathbf{B}(\omega)]=0,
$$

the positive roots of which determine the possible eigenfrequencies of the tip-sample for a given spatial configuration. As shown in a further section this result may be of practical interest for the calculation of the dispersion energy. Now for going further, it is necessary to specify more precisely the form of the tensor $\mathbf{S}$ appearing in the matrix $\mathbf{B}(\omega)$.

\section{DESCRIPTION OF THE SURFACE}

The propagator $\mathbf{S}\left(\mathbf{r}, \mathbf{r}^{\prime}, \omega\right)$ called also field susceptibility allows to know how a dipolar source field is modified at the immediate proximity of the surface of a solid. This feature can be clearly expressed by the linear relation (1). In the framework of a quantum description of the matter this quantity may be derived from the potential susceptibility $K_{s}$ of the solid (cf. Ref. 48). Another possibility lies in the research of the response field of the surface to a fluctuating dipole mo-

$$
\mathbf{F}_{0}(\omega)=\left[\mathbf{E}_{0}\left(\mathbf{R}_{1}, \omega\right), \mathbf{E}_{0}\left(\mathbf{R}_{2}, \omega\right), \ldots, \mathbf{E}_{0}\left(\mathbf{R}_{n}, \omega\right)\right] .
$$

Relation (2) becomes then

$$
\mathbf{F}(\omega)=\mathbf{F}_{0}(\omega)+\mathbf{B}(\omega) \cdot \mathbf{F}(\omega),
$$

where $\mathbf{B}(\omega)$ represents a $(3 n \times 3 n)$ matrix given by

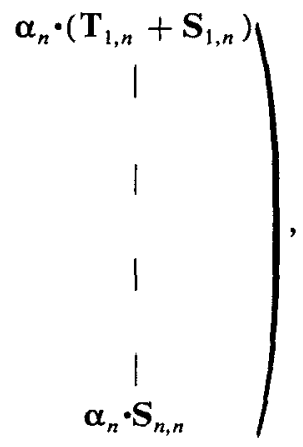

ment. In the particular case of a solid (dielectric or metallic) limited by a perfectly planar surface, various theoretical methods have been developed for building this tensor. The analytical form of this response function depends on the nature of the surface over interest. For example, to treat a given $(a, b, c)$ face of an ionic crystal it is more convenient to use a discrete atomic representation of the solid structure. ${ }^{50}$ Conversely, when one deals with a metal surface this quantity may be modeled from a continuous approach in order to take account into the delocalized character of the response of the free electrons. In this section we calculate such a propagator near a corrugated surface. Our approach is based on that of Marvin and Toigo. ${ }^{51}$ It is also closely related to the treatment of Rahman and Maradudin. ${ }^{52}$

\section{A. The source field of a dipole moment}

From the Maxwell equations we can write

$$
\begin{aligned}
\left(\Delta+k_{0}^{2}\right) \mathbf{E}(\mathbf{r}, \omega)= & -4 \pi\left\{k_{0}^{2} \mathbf{m}(\omega)+\nabla[\nabla \cdot \mathbf{m}(\omega)]\right\} \\
& \times \delta\left(\mathbf{r}-\mathbf{r}_{a}\right),
\end{aligned}
$$

where $\mathbf{r}_{a}$ represents the vector position of the dipole. The nonhomogeneous solution of Eq. (13) is ${ }^{53}$

$$
\begin{aligned}
\mathbf{E}_{m}(\mathbf{r}, \omega)= & \left\{k_{0}^{2} \mathbf{m}(\omega)+\nabla[\mathbf{m}(\omega) \cdot \nabla]\right\} \\
& \times \exp \left[i k_{0}\left|\mathbf{r}-\mathbf{r}_{a}\right|\right]\left|\mathbf{r}-\mathbf{r}_{a}\right|^{-1},
\end{aligned}
$$

where, due to the symmetry of the problem, the Green function

$$
G_{0}\left(\mathbf{r}-\mathbf{r}_{a}\right)=\exp \left[i k_{0}\left|\mathbf{r}-\mathbf{r}_{a}\right|\right]\left|\mathbf{r}-\mathbf{r}_{a}\right|^{-1}
$$

must be expanded in a Weyl series. ${ }^{53}$ One obtains then

$$
\begin{aligned}
\mathbf{E}_{m}(\mathbf{r}, \omega)= & \frac{i}{2 \pi} \iint \frac{d \mathbf{k}}{w_{0}}\left\{k_{0}^{2} \mathbf{m}(\omega)+\nabla[\mathbf{m}(\omega) \cdot \nabla]\right\} \\
& \times \exp \left[i \mathbf{k}_{0} \cdot\left(\mathbf{I}-\mathbf{I}_{a}\right)+i w_{0}\left|z-z_{a}\right|\right]
\end{aligned}
$$

with $\mathbf{r}=(\mathbf{l}, z), \mathbf{r}_{a}=\left(\mathbf{l}_{a}, z_{a}\right)$, and

$$
w_{0}^{2}=k_{0}^{2}-k^{2} \text {. }
$$




\section{B. Perfectly planar surface}

Let us consider a planar surface at $z=0$. We assume that the medium occupied just below this surface is characterized by a complex frequency-dependent dielectric constant $\epsilon(\omega)$. In the following this quantity will be assumed isotropic. The retarded response field of the surface to the excitation $\mathbf{m}(\omega)$ can be obtained from standard calculations involving appropriate boundary conditions at the surface $z=0$ :

$$
\begin{aligned}
\mathbf{E}_{r}(\mathbf{r}, \omega)= & \frac{i}{2 \pi} \int d \mathbf{k} f\left(\mathbf{r}, \mathbf{r}_{a}, \omega\right)\left\{\Delta _ { p } \mathbf { K } \left[k m_{z}(\omega)\right.\right. \\
& +\frac{w_{0} k_{y}}{k} m_{y}(\omega)+\frac{w_{0} k_{x}}{k} m_{x}(\omega) \\
& \left.-\Delta_{s} \mathbf{K}^{\prime}\left[\frac{k_{x}}{k} m_{y}(\omega)-\frac{k_{y}}{k} m_{x}(\omega)\right]\right\},
\end{aligned}
$$

where

$$
\mathbf{N}(\mathbf{k}, \omega)=\left(\begin{array}{cc}
\frac{\Delta_{p} w_{0} k_{x}^{2}}{k^{2}}-\frac{\Delta_{s} k_{0}^{2} k_{y}^{2}}{w_{0} k^{2}} & \frac{k_{x} k_{y}}{k^{2}}\left[\Delta_{p} w_{0}+\frac{\Delta_{s} k_{0}^{2}}{w_{0}}\right] \\
\frac{k_{x} k_{y}}{k^{2}}\left[\Delta_{p} w_{0}+\frac{\Delta_{s} k_{0}^{2}}{w_{0}}\right] & \frac{\Delta_{p} w_{0} k_{y}^{2}}{k^{2}}-\frac{\Delta_{s} k_{0}^{2} k_{x}^{2}}{w_{0}} \\
-\Delta_{p} k_{x} & -\Delta_{p} k_{y}
\end{array}\right.
$$

In this last equation the retarded character of the information is contained in the factors $w$ and $w_{0}$. When the light velocity $c \rightarrow \infty$ one recovers the expression of $S$ in the electrostatic limit. ${ }^{43}$

\section{Corrugation effects}

The general problem of the electromagnetic response of rough surfaces has been the object of many investigations. This problem has been treated by using integral equation and perturbative techniques in Ref. 52. In this context Rahman and Maradudin ${ }^{52}$ have calculated the response field to a point charge located near a rough dielectric vacuum interface. Corrections to the results for a perfectly planar surface were obtained in terms of Fourier transform of the corrugation function limiting the surface. In this part we show how it is possible to include such effects in our approach. We will thus define a corrugation propagator labeled $\widetilde{\mathbf{S}}\left(\mathbf{r}, \mathbf{r}^{\prime}, \omega\right)$ taking into account the real profile of the surface. Since in SNOM and in AFM the detection of surface roughness must be performed at a very small distance from the surface a nonretarded expression for $\widetilde{\mathbf{S}}\left(\mathbf{r}, \mathbf{r}^{\prime}, \omega\right)$ will be sufficient. Within this approximation the research of $\widetilde{\mathbf{S}}$ includes the following well known steps:

-Expansion of electric potential in plane waves.

-Application of boundary conditions for the electric

$$
\begin{aligned}
& \mathbf{K}=\left(k_{x} / k, k_{y} / k,-k / \omega_{0}\right), \\
& \mathbf{K}^{\prime}=\left(k_{0}^{2} / w_{0}\right)\left(-k_{y} / k, k_{x} / k, 0\right),
\end{aligned}
$$

and

$$
f\left(\mathbf{r}, \mathbf{r}_{a}, \omega\right)=\exp \left[i \mathbf{k} \cdot\left(\mathbf{l}-\mathbf{l}_{a}\right)+i w_{0}\left(z+z_{a}\right)\right] .
$$

Moreover, in Eq. (18) the two reflection coefficients are defined by

$$
\Delta_{p}=\frac{w-\epsilon(\omega) w_{0}}{w+\epsilon(\omega) w_{0}} ; \quad \Delta_{s}=\frac{w-w_{0}}{w+w_{0}},
$$

with

$$
w=\left[\epsilon(\omega) k_{0}^{2}-k^{2}\right]^{1 / 2} .
$$

Now by identifying Eqs. (18) and (1), one gets the dipolar propagator of the planar surface

$$
\mathbf{S}\left(\mathbf{r}, \mathbf{r}^{\prime}, \omega\right)=\frac{i}{2 \pi} \int d \mathbf{k} f\left(\mathbf{r}, \mathbf{r}^{\prime}, \omega\right) \mathbf{N}(\mathbf{k}, \omega)
$$

where $\mathbf{N}$ represents a second rank tensor which contains the dynamical information about the surface

$$
\left.\begin{array}{c}
\Delta_{p} k_{x} \\
\Delta_{p} k_{y} \\
-\frac{\Delta_{p} k^{2}}{w_{0}}
\end{array}\right)
$$

potential and the induction vector at the surface $z=\zeta(1)$.

-Perturbative resolution of the integral equation connecting various Fourier amplitude of the problem.

After some algebra the response potential of the surface appears as a sum of two contributions

$$
\phi_{r}(\mathbf{r}, \omega)=\bar{\phi}_{r}(\mathbf{r}, \omega)+\tilde{\phi}_{r}(\mathbf{r}, \omega)
$$

with, for the continuous part

$$
\begin{aligned}
\bar{\phi}_{r}(\mathbf{r}, \omega)= & \frac{1}{2 \pi}\left(\frac{\epsilon(\omega)-1}{\epsilon(\omega)+1}\right) \int k^{-1} d \mathbf{k} \mathbf{m}(\omega) \cdot \mathbf{Q} \\
& \times \exp \left[i \mathbf{k} \cdot\left(\mathbf{I}-\mathbf{I}_{\mathbf{z}}\right)-k\left(z+z_{u}\right)\right]
\end{aligned}
$$

and for the corrugation contribution

$$
\begin{aligned}
\tilde{\phi}_{r}(\mathbf{r}, \omega)= & \frac{1}{4 \pi^{3}} \frac{[\epsilon(\omega)-1]}{[\epsilon(\omega)+1]^{2}} \int d \mathbf{k} \int d \mathbf{k}^{\prime} \frac{\mathbf{m}(\omega) \cdot \mathbf{Q}^{\prime}}{k k^{\prime}} \\
& \times\left[\epsilon(\omega) k k^{\prime}+\mathbf{k} \cdot \mathbf{k}^{\prime}\right] \xi\left(\mathbf{k}-\mathbf{k}^{\prime}\right) \\
& \times \exp \left[i \mathbf{k} \cdot\left(\mathbf{1}-\mathbf{I}_{a}\right)-k\left(z+z_{a}\right)\right]
\end{aligned}
$$

with

$$
\mathbf{Q}=(i \mathbf{k}, k) \quad \mathbf{Q}^{\prime}=\left(i \mathbf{k}^{\prime}, k^{\prime}\right)
$$

and $\xi(\mathbf{q})$ represents the 2D Fourier transform of $\xi(1)$ :

$$
\xi(\mathbf{q})=\int d \mathbf{l} \exp (-i \mathbf{q} \cdot \mathbf{l}) \xi(\mathbf{1})
$$


The propagator $\widetilde{\mathbf{S}}\left(\mathbf{r}, \mathbf{r}^{\prime}, \omega\right)$ may be easily derived from Eq. (27). One finds

$$
\begin{aligned}
\widetilde{\mathbf{S}}\left(\mathbf{r}, \mathbf{r}^{\prime}, \omega\right)= & \frac{1}{4 \pi^{3}} \frac{[\epsilon(\omega)-1]}{[\epsilon(\omega)+1]^{2}} \iint d \mathbf{k} d \mathbf{k}^{\prime} \\
& \times \exp \left(i \mathbf{k} \cdot \mathbf{l}-i \mathbf{k} \cdot \mathbf{l}^{\prime}-k z+k^{\prime} z^{\prime}\right) \\
& \times\left\{\frac{\epsilon(\omega) k k^{\prime}+\mathbf{k} \cdot \mathbf{k}^{\prime}}{k k^{\prime}}\right\} \xi\left(\mathbf{k}-\mathbf{k}^{\prime}\right) \mathbf{Q}^{*} \mathbf{Q}^{\prime} ;
\end{aligned}
$$

the tensorial character of this response function is contained in the symbol $\mathbf{Q}^{*} \mathbf{Q}^{\prime}$. Note that this result corresponds to the first order contribution with respect to $\xi(\mathbf{q})$. Higher order terms including nonlinear contributions with respect to $\xi(\mathbf{q})$ have been neglected.

\section{TIP-SAMPLE DISPERSIVE FORCE}

Dispersion forces play a dominant role in the interaction between neutral material bodies. It is well known that the existence of such long range forces can be inferred from the correlations between the fluctuations of the charge densities inside the two partners. ${ }^{54-56}$ In the last decades many theoretical methods have thus been developed for explaining experimental data obtained by measuring the van der Waals force between quasiplanar surfaces. Theoretical expressions of this energy are available for specific geometries only ${ }^{55,56}$ (sphere-sphere, sphere-plane, cylinder-plane,...) and little work ${ }^{23}$ has been devoted to arbitrary configurations such as tip-sample system considered in this paper. The object of this section is twofold. First, a general expression of the tipsample dispersive force relevant in AFM will be derived from the self-consistent method developed in previous sections. Application to specific systems will be discussed in a second part.

\section{A. The interaction energy}

The knowledge of the dispersion equation (12) is sufficient for determining the free energy of interaction ${ }^{55}$

$$
V_{d}\left(\mathbf{R}_{0}\right)=k_{B} T \sum_{\xi_{q}}^{\prime} \log D\left(i \xi_{q}\right),
$$

where $T$ represents the absolute temperature, $\xi_{n}$ $=2 \pi n k_{B} T / \hbar$, and $q$ is a positive integer. The prime on the summation indicates that the term for $(q=0)$ must be weighted with a factor $1 / 2$. After replacing the Eq. (12) into Eq. (31) the energy may be expressed as

$$
V_{d}\left(\mathbf{R}_{0}\right)=k_{B} T \sum_{\xi_{q}}^{\prime} \log \left\{\operatorname{det}\left[\mathbf{I}-\mathbf{B}\left(i \xi_{q}\right)\right]\right\}
$$

For standard interatomic distances between each atom inside the tip and for tip-sample separation chosen far beyond the repulsive regime, every matrix element of $\mathbf{B}(\omega)$ remains small with respect to the unit. It is then possible to perform the following expansion:

$$
V_{d}\left(\mathbf{R}_{0}\right)=-k_{B} T \sum_{\xi_{q}}^{\prime} \sum_{m=1}^{\infty} \frac{1}{m} \operatorname{Tr}\left[\mathbf{B}^{(m)}\left(i \xi_{q}\right)\right],
$$

in which $\mathbf{B}^{(m)}(\omega)$ represents the matrix product of $m$ th order.

It may be seen that the first term ( $m=1)$ corresponds to the direct coupling between each element inside the tip and the surface. This term gives the main contribution to the dispersive energy. From Eq. (9), it may be expressed as

$$
\begin{aligned}
V_{d}^{(1)} & \left(R_{0}\right) \\
& =-k_{B} T \sum_{\xi_{q}}^{\prime} \sum_{i=1}^{n} \boldsymbol{\alpha}_{i}\left(i \xi_{q}\right): \mathbf{S}\left(\mathbf{R}_{0}+\mathbf{r}_{i}, \mathbf{R}_{0}+\mathbf{r}_{i}, i \xi_{q}\right),
\end{aligned}
$$

where $\mathbf{r}_{i}=\mathbf{R}_{i}-\mathbf{R}_{0}$ represents the position of the $i$ th atom with respect to the apex of the tip located at $\mathbf{R}_{0}=\left(0,0, Z_{0}\right)$. As expected, this equation does not take account of the many body effects inside the detector. Such contributions are included in higher order terms $(m>1)$. Finally, let us note that in Eq. (33) and (34) retardation and corrugations effects can be introduced by using Eqs. (23) and (30).

\section{B. Connection with substrate mediated energy (SME) of two adsorbed atoms}

When a pair of atoms or molecules is placed near a surface, a supplementary contribution called substrate-mediated energy must be taken into account. The study of such an alteration of the magnitude of the interatomic potential has been the subject of many theoretical investigations. ${ }^{47,57-59}$ In a recent contribution, ${ }^{57} \mathrm{it}$ is shown that a discrete description of the substrate can increase the magnitude of SME occurring between rare gas atoms with respect to the result obtained through a continuum approach. The aim of this part is to show that our general result [Eq. (33)] contains SME as a special case. When the multiatomic tip reduces to only two atoms of polarizability $\alpha_{1}(\omega)$ and $\alpha_{2}(\omega)$ located at the positions $\mathbf{R}_{1}$ and $\mathbf{R}_{2}$ the dynamical matrix $\mathbf{B}(\omega)$ becomes

$$
\mathbf{B}\left(\mathbf{R}_{1}, \mathbf{R}_{2}, \omega\right)=\left[\begin{array}{llllll}
\alpha_{1} S_{11}^{x x} & \alpha_{1} S_{11}^{x y} & \alpha_{1} S_{11}^{x z} & \alpha_{2} H_{12}^{x x} & \alpha_{2} H_{12}^{x y} & \alpha_{2} H_{12}^{x z} \\
\alpha_{2} S_{11}^{y x} & \alpha_{1} S_{11}^{y y} & \alpha_{1} S_{11}^{y z} & \alpha_{2} H_{12}^{y x} & \alpha_{2} H_{12}^{y y} & \alpha_{2} H_{12}^{y z} \\
\alpha_{1} S_{11}^{z x} & \alpha_{1} S_{11}^{z y} & \alpha_{1} S_{11}^{z z} & \alpha_{2} H_{12}^{z x} & \alpha_{2} H_{12}^{z y} & \alpha_{2} H_{12}^{z z} \\
\alpha_{1} H_{21}^{x x} & \alpha_{1} H_{21}^{x y} & \alpha_{1} H_{21}^{x z} & \alpha_{2} S_{22}^{x x} & \alpha_{2} S_{22}^{x y} & \alpha_{2} S_{22}^{x z} \\
\alpha_{1} H_{21}^{y x} & \alpha_{1} H_{21}^{y y} & \alpha_{1} H_{21}^{y z} & \alpha_{2} S_{22}^{y x} & \alpha_{2} S_{22}^{y y} & \alpha_{2} S_{22}^{y z} \\
\alpha_{1} H_{21}^{z x} & \alpha_{1} H_{21}^{z y} & \alpha_{1} H_{21}^{z z} & \alpha_{2} S_{22}^{z x} & \alpha_{2} S_{22}^{z y} & \alpha_{2} S_{22}^{z z}
\end{array}\right]
$$


with

$$
H_{i j}=S_{i j}+T_{i j} \text {. }
$$

In this case the second order contribution of Eq. (33)

$$
\begin{aligned}
& V_{d}^{(2)}\left(\mathbf{R}_{1}, \mathbf{R}_{2}\right) \\
& \quad=-\frac{k_{B} T}{2} \sum_{\xi_{q}}^{\infty} \operatorname{Tr}\left[\mathbf{B}\left(\mathbf{R}_{1}, \mathbf{R}_{2}, i \xi_{q}\right) \cdot \mathbf{B}\left(\mathbf{R}_{1}, \mathbf{R}_{2}, i \xi_{q}\right)\right]
\end{aligned}
$$

leads to

$$
\begin{aligned}
V_{d}^{(2)}\left(\mathbf{R}_{1}, \mathbf{R}_{2}\right)= & -\frac{k_{B} T}{4}\left(1+P_{12}\right) \sum_{\xi_{q}}^{\infty} \operatorname{Tr}\left[\boldsymbol{\alpha}_{1}\left(i \xi_{q}\right)\right. \\
& \left.\cdot \mathbf{T}\left(\mathbf{R}_{1}, \mathbf{R}_{2}\right) \cdot \boldsymbol{\alpha}_{2}\left(i \xi_{q}\right) \cdot \mathbf{S}\left(\mathbf{R}_{2}, \mathbf{R}_{1}, i \xi_{q}\right)\right] \\
& -\frac{k_{B} T}{4} \sum_{\xi_{q}}^{\infty} \operatorname{Tr}\left[\boldsymbol{\alpha}_{1}\left(i \xi_{q}\right) \cdot \mathbf{S}\left(\mathbf{R}_{1}, \mathbf{R}_{2}, i \xi_{q}\right)\right. \\
& \left.\cdot \boldsymbol{\alpha}_{2}\left(i \xi_{q}\right) \cdot \mathbf{S}\left(\mathbf{R}_{2}, \mathbf{R}_{1}, i \xi_{q}\right)\right],
\end{aligned}
$$

which recovers the well known results of SME given in Refs. 58 and 59. In Eq. (37) $P_{12}$ defines the permutation operator. Thus this connection brings some consistency between our self-consistent approach and other theories.

\section{Application to specific systems}

Calculation of the magnitude of dispersive force derived from $V_{d}$ [Eq. (33)] requircs the knowledge of $\alpha_{i}(i \xi)$ and $\epsilon(i \xi)$ both evaluated at imaginary frequency. In this section we present a numerical study based on the relation (33) for systems of experimental interest: a diamond tip interacting with a planar dielectric surface (mica) and metallic substrates.

\section{The detector}

It will be modeled as an ensemble of $n$ carbon atoms of dipolar polarizability

$$
\alpha(i \xi)=\frac{\alpha(0) \omega_{0}^{2}}{\omega_{0}^{2}+\xi^{2}}
$$

where $\alpha(0)$ and $\omega_{0}$ label the static polarizability and the effective frequency associated to carbon atoms of the probe. These two quantities may be connected to the $C_{6}$ dispersion coefficient between a pair of atoms. Note that a more involved description could be introduced through an expansion of the dynamical polarizability expressed in terms of Cauchy moments. Such improvements will be included in a further calculation.

\section{The substrate}

It is well known that dielectric data may be obtained from electron energy loss spectroscopy and by analysis of reflexivity measurements. In a theoretical study of van der Waals forces between mica and quartz plates, Chan and Richmond $^{\text {s0 }}$ have shown how, by using such data, it was possible to build a dielectric function $\epsilon(i \xi)$ available for dispersive force computation. For mica and quartz $\epsilon(i \xi)$ may be expressed as a function of three parameters $\epsilon_{\mathrm{uv}}, \omega_{s}$, and $\eta$ :
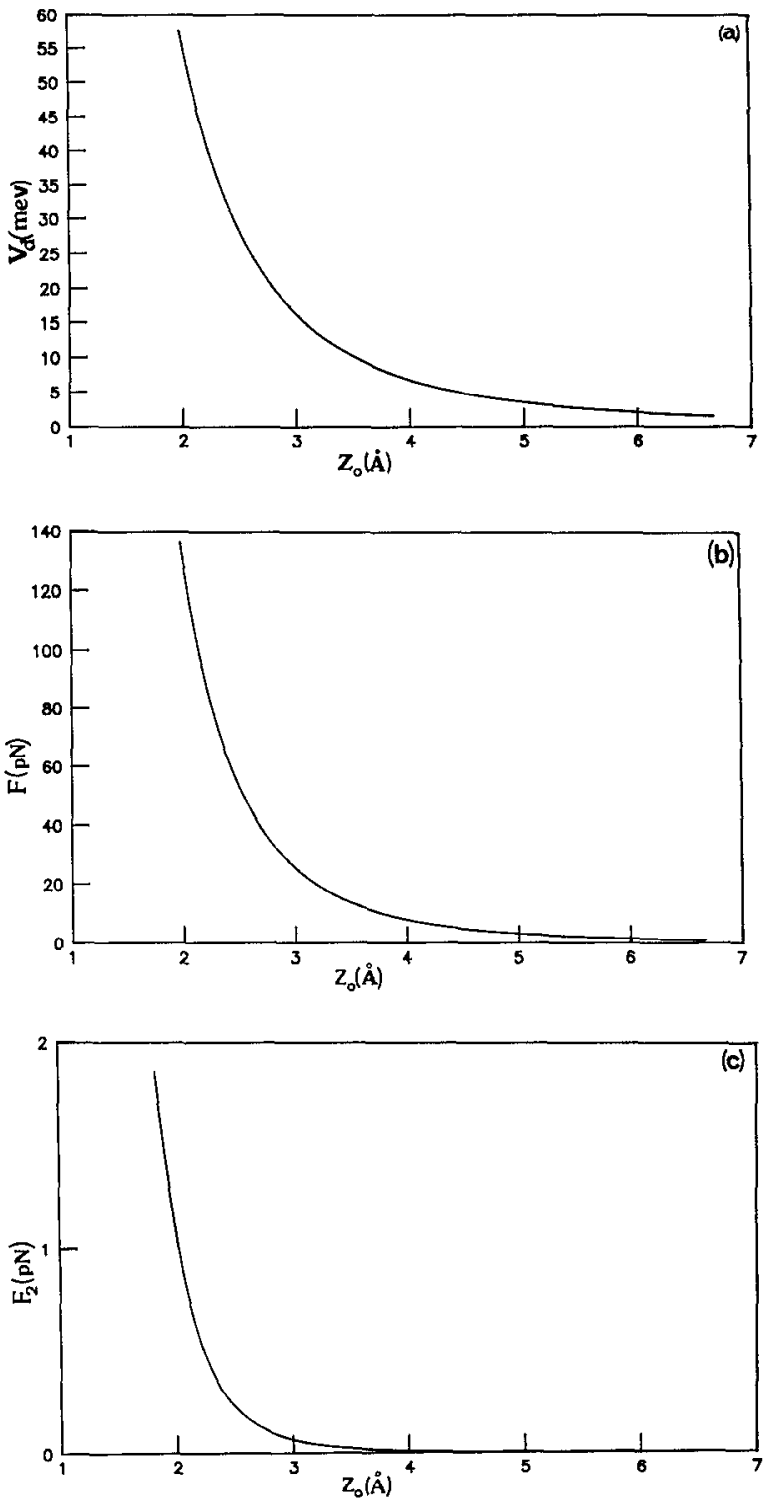

FIG. 3. Interaction between a monoatomic tip and a mica sample: (a) dispersion energy as a function of the approach distance; (b) the total attractive dispersion force; (c) the second order contribution to the dispersion force as given in Eq. (33).

$$
\epsilon(i \xi)=1+\frac{\epsilon_{\mathrm{uv}}-1}{1+\left(\xi / \omega_{s}\right)^{2}+(\xi / \eta)} .
$$

From relations (33), (38), and (39) we have calculated the magnitude of the interaction force between a diamond tip placed near a dielectric (mica) or a metallic ( $\mathrm{Au}, \mathrm{Ag}$, $\mathrm{Cu}$ ) surface. For the metallic case we have used the data of Vidali and $\mathrm{Cole}^{61}$ in which the reflection coefficient of the surface is based on the knowledge of two fitted parameters $\left(g_{0}\right.$ and $\left.\omega_{s}\right)$. The corresponding data used for this numerical study are given in Refs. 22, 61, and 62 and results obtained from these values are presented in Figs. 3 to 5 for various size of the probe. In Fig. 3, the force and the energy experienced by a monoatomic tip placed above a planar mica sample are plotted as a function of the approach distance $Z_{0}$. At about 3 $\AA$ from the surface, the energy and the corresponding force 


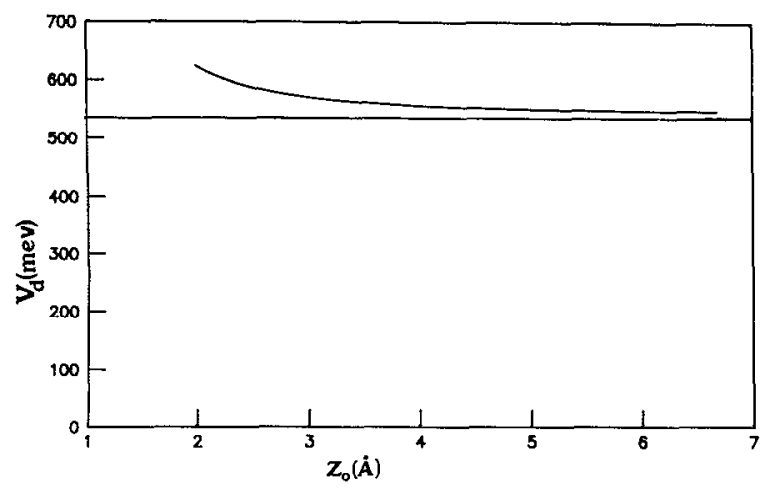

FIG. 4. Magnitude of the dispersion energy for a tip formed of four carbon atoms interacting with a mica sample. The straight line corresponds to the dispersive cohesive energy of the isolate tip.

are found equal to $15 \mathrm{meV}$ and to $0.25 \times 10^{-10} \mathrm{~N}$, respectively [cf. Figs. 3 (a) and 3 (b) ]. Moreover, on the curve given in Fig. 3(c), it may be seen that the second order contribution force $F_{2}\left(Z_{0}\right)$ corresponding to multiple reflections between the tip and the sample introduces a weak contribution to the total force. This result is consistent with the fast spatial dependence of such terms in which the propagator of the surface appears at a power $m>1$. Consequently, this term becomes negligible at distances greater than $2.5 \AA$.

Figure 4 represents the behavior of the dispersion energy when a four-atom tip is placed in front of a mica sample. In this case, one remarks that $V_{d}\left(Z_{0}\right)$ does not vanish when the tip-sample separation increases. In fact, the present formalism takes into account the whole van der Waals energy of the system. Consequently, when $Z_{0} \rightarrow \infty$, the asymptotic value of $V_{d}$ corresponds to the van der Waals cohesive energy of the atoms forming the tip.

Figures (5) and (6) give the magnitude of the dispersion force experienced by a multiatomic tip ( 20 carbon atoms). In the case of mica substrate the force reaches $0.4 \times 10^{-10} \mathrm{~N}$ at an approach distance $Z_{0}=3 \AA$ while for noble metal surfaces (Fig. 6) one finds about $0.58 \times 10^{-10} \mathrm{~N}$ at the same distance. Such magnitude orders are in good agreement with those measured in AFM working in attrac-

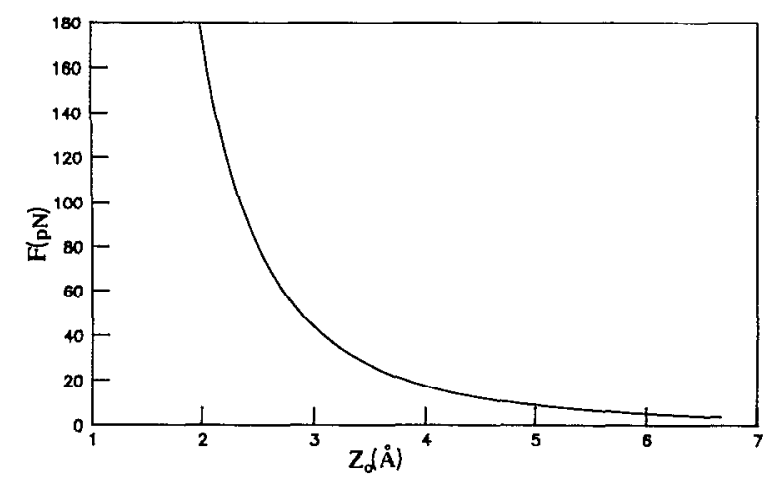

FIG. 5. Dispersion force experienced by a tip formed of 20 carbon atoms placed near a mica sample.

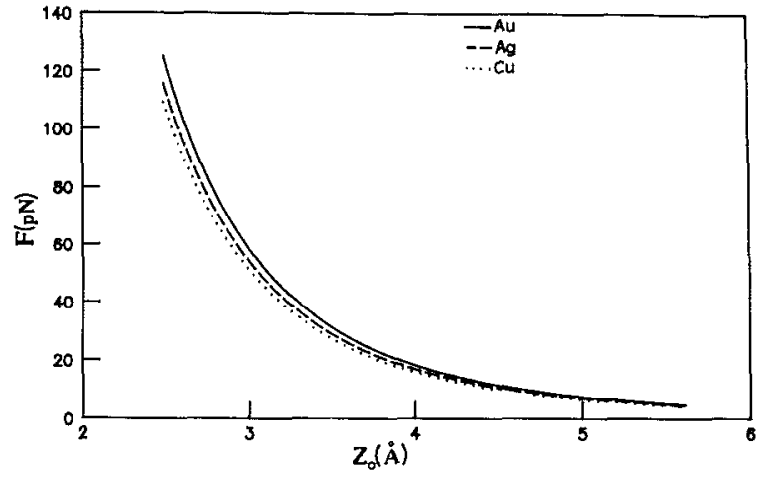

FIG. 6. Magnitude of the dispersion force of a tip formed of 20 carbon atoms interacting with noble metals.

tive regime. Note that a detailed numerical study of this problem by introducing corrugation effects from corrugation propagator $\widetilde{S}$ given in Sec. III $\mathrm{C}$ is left for a forthcoming paper.

\section{COUPLED MODES AND SNOM}

Recent experimental studies based on the use of a local optically transparent detector have allowed to image various forms of subwavelength structures lying on dielectric surfaces. ${ }^{24-28}$ A complete review of this field has been recently proposed. ${ }^{24}$ In this work, it is indicated that theoretical descriptions of SNOM properties, such as resolution and contrast, need models for describing the whole light-matter interaction between a tip and a corrugated surface. Thus very recently a self-consistent treatment ${ }^{30}$ in which the probe is approximated as a polarizable sphere has provided various theoretical images of nanometric infinite tracks.

The aim of this section is to show that the present description allows us to remove the spherical approximation used in Ref. 30 by including the real profile of the tip from the discrete scheme given in Sec. II. Moreover, from above relations we will analyze the role played by the coupled modes of the tip-sample system on the efficiency of the near field-radiative field transfer. This discussion will be performed in the framework of the total reflection configuration introduced in SNOM by Reddick et al. ${ }^{25}$ and by Courjon et $a l .{ }^{26}$ In these devices, a transparent sample is illuminated so that total reflection occurs at the surface of the object. Note that our formalism could also be used for modeling other experimental configurations such as the one applied in reflection optical near field microscopy. This point will not be considered here.

Let us consider a plane wave falling on the boundary between two homogeneous media (air and glass interface). In the case of total reflection, the evanescent field in the halfspace $z>0$ is given by

$$
\mathbf{E}_{0}(\mathbf{r}, \omega)=\mathbf{E}_{0}(k, \theta) \exp \left(i \mathbf{K}^{t} \cdot \mathbf{r}\right)
$$

with

$$
\mathbf{E}_{0}(k, \theta)=\left[E_{0 x}(k, \theta), E_{0 y}(k, \theta), E_{0 z}(k, \theta)\right]
$$

and 


$$
\mathbf{K}^{t}=k_{0}\left[\sin \theta, 0, i\left(\sin ^{2} \theta-\sin ^{2} \theta_{L}\right)^{1 / 2}\right],
$$

where $\theta$ is the incident angle and $\theta_{L}$, the limit angle of refraction. In the absence of probe, the field distribution in any point $\mathbf{R}_{i}$ above the surface is given by the ( $\left.3 n\right)$ supervector $F_{0}(\omega)$ [Cf. Eq. (7)] defined by

$\mathbf{F}_{0}(\omega)=\mathbf{E}_{0}(\theta, k)\left[\exp \left(i \mathbf{K}^{t} \cdot \mathbf{R}_{1}\right), \ldots, \exp \left(i \mathbf{K}^{t} \cdot \mathbf{R}_{n}\right)\right]$.

The knowledge of this relation and Eq. (10) allows one to obtain the real field distribution experienced by each microscopic component of the detector. The general solution giving the effective field on each microsystem can then be written in the form

$E_{\beta}\left(\mathbf{R}_{j}, \omega\right)=\sum_{i, \alpha} \frac{A_{\alpha i, \beta j}(\omega)}{\operatorname{det}[\mathbf{I}-\mathbf{B}(\omega)]} E_{0 \alpha}(k, \theta) \exp \left(i \mathbf{K}^{t} \cdot \mathbf{R}_{i}\right)$

where the denominator is the determinant of the dynamical coupling matrix $[\mathbf{I}-\mathbf{B}(\omega)]$ and the numerator represents the cofactor of the $(\alpha i, \beta j)$ element of this matrix. Finally, from this relation it is easy to determine the scattered intensity of the radiative field inside the upper part of the tip. In a first step, one writes the field emitted by the $(j)$ th component at a distance $\mathbf{R}_{0 b}$ from the tip extremity

$$
\begin{aligned}
\mathbf{E}_{j}^{(r)}\left(\mathbf{R}_{0 b}, \omega\right)= & \mathbf{T}_{1}\left(\mathbf{R}_{0 b}-\mathbf{R}_{j}\right) \cdot \boldsymbol{\alpha}_{j}(\omega) \cdot \mathbf{E}\left(\mathbf{R}_{j}, \omega\right) k_{0}^{2} \\
& \times \exp \left(-i k_{0}\left|\mathbf{R}_{0 b}-\mathbf{R}_{j}\right|\right)
\end{aligned}
$$

which leads to an intensity proportional to

$$
\begin{aligned}
I \simeq & \frac{\omega^{4}}{c^{4}} \frac{1}{|\operatorname{det}[\mathbf{I}-\mathbf{B}(\omega)]|^{2}} \\
& \times \sum_{\alpha} \mid \sum_{i, j} \sum_{\beta, \gamma} T_{1_{\alpha \beta}}\left(\mathbf{R}_{0 b}-\mathbf{R}_{j}\right) A_{\alpha i, \beta j}(\omega) \alpha_{j}(\omega) \\
& \times\left. E_{0 \gamma}(\theta, k) \exp \left(i \mathbf{K}^{t} \cdot \mathbf{R}_{i}\right)\right|^{2} .
\end{aligned}
$$

This quantity which is the relevant observable in the experiment situation described in Refs. 25 and 26 contains all the dynamical and structural informations about the surface and the tip through the properties of the matrix $B(\omega)$. Moreover, it may be seen on this relation that the efficiency of the tip-sample coupling depends on the relative magnitude of the eigenfrequencies $\omega_{c}$ with respect to the one of the external field. In the particular case where we deal with a metallic tip, metallic protuberance included in a dielectric stylus or a metallic substrate it is possible to excite in an efficient way a given mode $\omega_{c}$ with an optical field. Indeed, when

$$
\operatorname{Re}\{\operatorname{det}[\mathbf{I}-\mathbf{B}(\omega)]\}=0,
$$

the detected intensity exhibits a resonance for a given tipsample configuration. Such resonant effects have been recently observed by generating surface plasmons on a metallic detector placed near a solid. ${ }^{4}$ In the near field zone (at about $100 \mathrm{~nm}$ from the surface) extremely narrow resonances vs the approach distance were observed.

\section{CONCLUSION}

In this paper we have described a microscopic approach allowing a qualitative and quantitative discussion of physical mechanisms connected to local probe microscopy experiments (AFM, SNOM, - ) . In this context, we have shown that the coupled modes of the system govern different observables measured in experimental situations (dispersion energy, scattered intensity, $\cdots$ ). These normal modes are derived by including all dipolar correlations inside the detector and the surface (plane or corrugated). Consequently, this formalism appears as a convenient tool for modeling nanometer size detector of arbitrary shape. Another advantage of the method lies in the use of propagators. Such a formulation thus opens a direct way of investigating various types of surfaces through the appropriate propagators available in literature [nonlocal metallic surfaces, $(a, b, c)$ faces of an ionic crystal, semiconductor $\cdots$ ]. For example, corrugation effects introduced in our formalism through the tensor $\widetilde{\mathbf{S}}$ allow to include the real profile of a given surface without formal difficulty. This feature will be useful for studying the image shapes obtained in reflection near field optical microscopy. Finally, as it stands, the present description could be generalized in more involved situations (two dimensional lattice of metallic spheres deposited on a planar substrate, study of the influence of higher order multipolar moments in the framework of the spherical tensor analysis,...).

'G. Binnig, H. Rohrer, Ch. Gerber, and E. Weibel, Phys. Rev. Lett. 49, 57 (1982).

2D. W. Pohl, W. Denk, and M. Lanz, Appl. Phys. Lett. 44, 651 (1984).

${ }^{3}$ G. Binnig, C. F. Quate, and Ch. Gerber, Phys. Rev. Lett. 56, 930 (1986).

${ }^{4}$ U. Ch. Fischer and D. W. Pohl, Phys. Rev. Lett. 62, 458 (1989).

${ }^{5} \mathrm{D}$. Courjon (private communication).

'J. H. Coombs, J. K. Gimzewski, B. Reihl, J. K. Sass, and R. R. Shlittler, J. Microsc. 152, 325 (1988).

${ }^{7}$ C. M. Mate, R. Erlandsson, G. M. McClelland, and S. Chiang, J. Vac. Sci. Technol. A 6, 575 (1988).

${ }^{8}$ J. Schneir, O. Marti, G. Remmers, D. Glaser, R. Somnenfeld, B. Drake, P. K. Hansma, and V. Elings, J. Vac. Sci. Technol. 6, 283 (1988).

'O. Marti, B. Drake, S. Gould, and P. K. Hansma, J. Vac. Sci. Technol. 6, 287 (1988).

I'U. Dürig, J. K. Gimzewski, and D. W. Pohl, Phys. Rev, Lett, 57, 2403 (1986).

"'S. L. Tang, J. Bokor, and R. H. Storz, Appl. Phys. Lett. 52, 188 (1988).

${ }^{12}$ D. W. Abraham, C. C. Williams, and H. K. Wickramasinghe, Appl. Phys. Lett. 53, 1446 (1988).

${ }^{13}$ S. A. C. Gould, K. Burke, and P. K. Hansma, Phys. Rev. B 40, 8 (1989).

${ }^{14}$ H. K. Wickramasinghe, J. Vac. Sci. Technol. A 8, 363 ( 1990).

${ }^{15}$ I. P. Batra and S. Ciraci, J. Vac. Sci. Technol. A 6, 313 (1988).

${ }^{16}$ F. F. Abraham, I. P. Batra, and S. Ciraci, Phys. Rev. Lett. 60, 1314 (1988)

${ }^{17}$ S. Ciraci, A. Baratoff, and I. P. Batra, Phys. Rev. B 41, 2763 (1990).

${ }^{18}$ U. Landman, W. D. Luedtke, and A. Nitzan, Surf. Sci. 210, L177 (1989).

${ }^{19}$ S. A. C. Gould, K. Burke, and P. K. Hansma, Phys. Rev. B 40, 5363 (1990).

${ }^{20}$ C. Girard, D. Van Labeke, and J. M. Vigoureux, Phys. Rev. B 40, 12133 (1990).

${ }^{21}$ D. Van Labeke, B. Labani, and C. Girard, Chem. Phys. Lett. 162, 399 (1989).

${ }^{22}$ C. Girard, S. Maghezzi, and C. Van Labeke, Surf. Sci. 234, 181 (1990).

${ }^{23}$ U. Hartmann, Phys. Rev. B 42, 1541 (1990).

${ }^{24} \mathrm{D}$. W. Pohl, Advances in Optical and Electron Microscopy (Academic, London).

${ }^{25}$ R. Reddick, R. J. Warmack, and T. L. Ferrell, Phys. Rev. B 39, 767 (1989).

${ }^{26} \mathrm{D}$. Courjon, K. Sarayeddine, and M. Spajer, Opt. Commun. 71, 23 (1989).

${ }^{27}$ F. De Fornel, J. P. Goudonnet, L. Salomon, and E. Lesniewska, EC02 Opt. Storage Scan. Technol. 1139, 77 (1989). 
${ }^{28}$ D. Courjon, J. M. Vigoureux, M. Spajer, K. Sarayeddine, and S. Leblanc, Appl. Opt. 29, 3734 (1990).

${ }^{29}$ B. Labani, C. Girard, D. Courjon, and D. Van Labeke, J. Opt. Soc. Am. B 7, 936 (1990).

${ }^{30} \mathrm{C}$. Girard and D. Courjon, Phys. Rev. B 42, 9340 (1990).

${ }^{31}$ W. Denk and D. W. Pohl, Surf. Sci. (in press, 1990).

${ }^{32}$ J. Gersten and A. Nitzan, J. Chem. Phys. 73, 3023 (1980); 75, 1139 (1981).

${ }^{33}$ K. Araya and R. Zeyher, Phys. Rev. B 24, 1852 (1981).

${ }^{34}$ P. F. Liao, Surface Enhanced Raman Scattering, edited by R. K. Chang and T. E. Furtak (Plenum, New York, 1982), p. 379.

${ }^{35}$ P. K. Aravind and H. Metiu, J. Phys. Chem. 86, 5076 (1982); Surf. Sci. 124, 506 (1982).

${ }^{36}$ A. Wokaun, J. P. Gordon, and P. F. Liao, Phys. Rev. Lett. 48, 957 (1982).

${ }^{37}$ H. Metiu, Prog. Surf. Sci. 17, 153 (1984).

${ }^{38}$ H. Metiu and P. Das, Annu. Rev. Phys. Chem. 35, 507 (1984).

${ }^{39}$ G. W. Ford and W. H. Weber, Phys. Rep. 113, 195 (1984).

${ }^{40}$ H. Meier, A. Wokaun, and P. F. Liao, J. Opt. Soc. Am. B 2, 931 (1985).

${ }^{41}$ A. Wokaun, Mol. Phys. 56, 1 (1985).

${ }^{42}$ P. Barnickel and A. Wokaun, Mol. Phys. 67, 1355 (1989).

${ }^{43}$ A. D. McLachlan, Mol. Phys. 7, 381 (1964).

${ }^{44}$ B. Linder, Advan. Chem. Phys. 12, 225 (1967).
${ }^{45}$ B. Linder and D. A. Rabenold, Advan. Quant. Chem. 6, 203 (1972).

${ }^{46} \mathrm{G}$. Korzeniewski, T. Maniv, and $\mathrm{H}$. Metiu, Chem. Phys. Lett. 73, 212 (1980); J. Chem. Phys. 76, 1564 (1982).

${ }^{47}$ H. Metiu, J. Chem. Phys. 76, 1765 (1982).

${ }^{48}$ C. Girard, J. Chem. Phys. 85, 6750 (1986).

${ }^{49} \mathrm{C}$. Girard, S. Maghezzi, and F. Hache, J. Chem. Phys. 91, 5509 (1989).

${ }^{50} \mathrm{C}$. Girard and C. Girardet, J. Chem. Phys. 86, 6581 (1987).

${ }^{51}$ A. M. Marvin and F. Toigo, Phys. Rev. A 25, 782 (1982).

${ }^{52}$ T. S. Rahman and A. A. Maradudin, Phys. Rev. B 12, 504 (1980).

${ }^{53}$ G. S. Agarwal, Phys. Rev. A 11, 230 (1975).

${ }^{54}$ E. M. Lifshitz, Zh Ersp. Theor. Fiz. 29, 94 ( 1955) [Sov. Phys. -JETP 2, 73 (1956)].

${ }^{55} \mathrm{~J}$. Mahanty and B. W. Ninham, Dispersion Forces (Academic, London, 1976).

${ }^{56} \mathrm{D}$. Langbein, Theory of van der Waals Attraction (Springer, Berlin, 1976).

${ }^{57}$ P. Richmond and K. W. Sarkies, J. Phys. C 6, 401 (1973).

${ }^{58}$ S. Rauber, J. R. Klein, M. W. Cole, and L. W. Bruch, Surf. Sci. 123, 173 (1982).

${ }^{59} \mathrm{C}$. Girard and C. Girardet, Surf. Sci. 195, 173 (1988).

${ }^{6}$ D. Chan and P. Richmond, Proc. R. Soc. London Ser. A 353, 163 (1977).

${ }^{\circ} \mathrm{G}$. Vidali and M. W. Cole, Surf. Sci. 110, 10 (1981). 